\title{
VALUATION RINGS WITH ZERO DIVISORS
}

\author{
PATRICK H. KELLY AND MAX D. LARSEN ${ }^{1}$
}

ABstract. Manis has developed a valuation theory for commutative rings which extends valuation theory for fields. However his results do not extend the characterization of valuation rings as domains of maximal partial homomorphisms. In this note we show that Manis' theory also generalizes this aspect of valuation theory. Next we show that an overring $W$ of a valuation ring $V$ is not necessarily a valuation ring in any nice sense, but that $W$ is contained in a valuation ring which is a large quotient ring of $V$.

All rings considered will be commutative and have unity element. An element will be called regular if it is not a zero divisor and an ideal will be called regular if it contains a regular element. An overring of a ring $R$ is a ring between $R$ and its total quotient ring. For convenience $A \backslash B=\{x \in A \mid x \notin B\}$.

1. Maximal partial homomorphisms and valuation pairs. If $K$ is a ring, then by a valuation pair of $K$, we mean a pair $(V, P)$, where $V$ is a subring of $K$ and $P$ is a prime ideal of $V$, satisfying any of the following equivalent conditions [5, p. 193]:

(1) If $W$ is a subring of $K$ containing $V$ and if $M$ is a prime ideal of $W$ with $M \cap V=P$, then $V=W$.

(2) For all $x \in K \backslash V$, there exists $p \in P$ such that $p x \in V \backslash P$.

(3) There is a mapping $v$ from $K$ onto an ordered abelian group with symbol $\infty$ adjoined which satisfies $v(x y)=v(x)+v(y)$ and $v(x+y) \geqq \min \{v(x), v(y)\}$ for all $x, y \in K$ and such that

$$
V=\{x \in K \mid v(x) \geqq 0\} \text { and } P=\{x \in K \mid v(x)>0\} .
$$

In [2, Theorem 1] the equivalent conditions which define a valuation ring of a field include a condition involving maximal partial homomorphisms. We now establish this result for rings which allow zero divisors.

Presented to the Society, August 21, 1970 under the title Valuation pairs and maximal partial homomorphisms; received by the editors June 18, 1970 and, in revised form, August 10, 1970 and November 16, 1970.

AMS 1970 subject classifications. Primary 13A15.

Key words and phrases. Maximal partial homomorphism, valuation pair, overring of valuation ring.

1 During the writing of this paper, this author held a Senior Faculty Summer Fellowship from the Research Council of the University of Nebraska.

Copyright (C) 1971, American Mathematical Society 
Definition. Let $K$ be a ring and let $V$ be a subring of $K$. If $h$ is a homomorphism from $K$ into an algebraically closed field $L$ with domain $V, h$ is called a maximal partial homomorphism of $K$ if $h$ has no extension to a homomorphism from $K$ into $L$ with domain a subring of $K$ properly containing $V$.

This definition provides an alternative to the definition of place given by Harrison [4, p. 53].

Theorem 1. Let $K$ be a ring, let $V$ be a subring of $K$, and let $P$ be $a$ prime ideal of $V$. Then $(V, P)$ is a valuation pair of $K$ if and only if there exists an algebraically closed field $L$ and a maximal partial homomorphism $h$ from $K$ into $L$ with domain $V$ and kernel $P$.

Proof. Suppose $(V, P)$ is a valuation pair. Let $L$ be an algebraic closure of the quotient field of $V / P$. Let $h$ be the canonical homomorphism $V \rightarrow V / P$. Suppose $h$ has an extension $h^{\prime}: W \rightarrow L$ where $V \subset W \subseteq K$. If $x \in W \backslash V$, there exists $p \in P$ such that $x p \in V \backslash P$. Then $h^{\prime}(x p)=h(x p) \neq 0$, but $h^{\prime}(x p)=h^{\prime}(x) h^{\prime}(p)=0$. Hence $h$ is maximal.

Now suppose $(V, P)$ is not a valuation pair; then there is a ring $W$ with $V \subset W \subseteq K$ and a prime ideal $M$ of $W$ such that $M \cap V=P$. Let $L$ be an algebraically closed field and let $h$ be a maximal partial homomorphism from $K$ into $L$ with domain $V$ and kernel $P$. Then $V / P \subseteq L$. Consider $V / P$ a subring of $W / M$ and identify elements under the usual injection. If $W / M$ is algebraic over $V / P$ then the mapping $W \stackrel{\text { nat }}{\rightarrow} W / M \stackrel{\text { inj }}{\rightarrow} L$ extends $h$. Otherwise some $w+M$ is transcendental over $V / P$, where $w \in W \backslash V$. Define a mapping $h^{\prime}: V[w] \rightarrow L$ by $h^{\prime}\left(\sum_{i=0}^{n} b_{i} w^{i}\right)=h\left(b_{0}\right)$. To see that $h^{\prime}$ is well-defined, let $c=\sum_{i=0}^{n} b_{i} w^{i} \in V[w]$ be such that $c=0$. Then in $W / M$,

$$
\sum_{i=0}^{n}\left(b_{i}+M\right)(w+M)^{i}=0
$$

and $b_{i}+M=b_{i}+P \in V / P$. Therefore $b_{0} \in P$ and $h\left(b_{0}\right)=0$, and hence $h^{\prime}(c)=0$.

CoROllary. Let $K$ be a ring with subring $V$. Let $h$ be a maximal partial homomorphism of $K$ with domain $V$ into an algebraically closed field $L$. If $K^{\prime}$ is an overring of $K$, there is a maximal partial homomorphism $h^{\prime}$ of $K^{\prime}$ into $L$ which extends $h$. Further, if $x_{1}, x_{2}, \cdots, x_{n}$ are elements of $K^{\prime}$ such that $\sum a_{i_{1} i_{2} \cdots i_{n}} x_{1}^{i_{1}} x_{2}^{i_{2}} \cdots x_{n}^{i_{n}}=0$ implies all the $a_{i_{1} i_{2} \cdots i_{n}}$ are in the kernel of $h$, and if $a_{1}, a_{2}, \cdots, a_{n}$ are arbitrary elements of $L$, then $h^{\prime}$ can be chosen such that $h^{\prime}\left(x_{i}\right)=a_{i}$ for $i=1$, $2, \cdots, n$.

2. Valuation rings. For the remainder of this paper, if $(V, P)$ is a 
valuation pair of $K$ where $K$ is the total quotient ring of $V$, we will say simply that $(V, P)$ is a valuation pair.

If $P$ is a prime ideal in a ring $V$ with total quotient ring $K$, the large quotient ring $[3$, p. 56] of $V$ with respect to $P$ is

$$
V_{[P]}=\{x \in K \mid x s \in V \text { for some } s \in V \backslash P\} .
$$

If $(V, P)$ is a valuation pair, then $V=V_{[P]}$.

The next results show that a ring cannot form a valuation pair with two distinct regular prime ideals.

Proposition. Let $(V, P)$ be a valuation pair with $P$ regular and let $Q$ be a prime ideal of $V$. Then $V_{[Q]}=V$ if and only if $P \subseteq Q$.

Proof. If $P \subseteq Q$, then $V \subseteq V_{[Q]} \subseteq V_{[P]}=V$. Now suppose $P \nsubseteq Q$ and assume that $V=V_{[Q]}$. Let $r$ be a regular element of $P$; then $r \in Q$. If $p \in P \backslash Q$, then $p / r \notin V$, so there exists $s \in P$ such that $s p / r$ $\in V \backslash P$. Since $V=V_{[Q]}$ and $p \notin Q, s / r \in V$ and therefore $s p / r \in P$, a contradiction.

CoROllaRY. Let $V$ be a ring, $K$ its total quotient ring. Either $V=K$ or there exists at most one prime ideal $P$ of $V$ such that $(V, P)$ is a valuation pair of $K$. If such $P$ exists, it is regular if and only if $V \neq K$.

Proof. Suppose $V \neq K$ and $P, Q$ are prime ideals of $V$ with $(V, P)$ and $(V, Q)$ valuation pairs of $K$. Since $V \neq K$, there exists a regular element $x \in V$ with $x^{-1} \notin V$. Since $V_{[Q]}=V_{[P]}, x \in P \cap Q$, so $P$ and $Q$ are both regular. That $P=Q$ follows from the proposition.

Because of the above result, when $(V, P)$ is a valuation pair, we shall call $V$ a valuation ring.

3. Overrings of valuation rings. We show that overrings of valuation rings are not necessarily valuation rings in the nice way that they are for integral domains.

Theorem 2. Let $(V, P)$ be a valuation pair with $P$ regular. If for every overring $W$ of $V$ there exists an ideal $M$ of $W$ contained in $V$ such that $(W, M)$ is a valuation pair, then $P$ is a maximal ideal of $V$.

Proof. Let $b$ be a regular element of $P$ and let $a$ be any element in $V \backslash P$. Let $W=V[a / b]$ and let $M$ be the prime ideal given by the hypothesis. Then $M \subseteq P$ since $(V, P)$ being a valuation pair implies that $P \supseteq\{x \in V \mid x y \in V$ for some $y \notin V\}$. Then $b \notin M$ and $1 / b \in W$ since $(W, M)$ is a valuation pair. Thus there is a smallest positive integer $n$ and $c_{0}, c_{1}, \cdots, c_{n} \in V$ such that $c_{n} \neq 0$ and

$$
1 / b=\sum_{i=0}^{n} c_{i}(a / b)^{i}
$$


If $n \geqq 2$, multiplying by $b^{n-1}$ shows that $c_{n} a^{n-1}(a / b) \in V$. But since $a^{n-1} \in V \backslash P$ and $V=V_{[P]}$ this means that $c_{n}(a / b) \in V$, so

$$
1 / b=\sum_{i=0}^{n-2} c_{i}(a / b)^{i}+\left(c_{n-1}+c_{n}(a / b)\right)(a / b)^{n-1},
$$

a contradiction. Therefore $1 / b=c_{0}+c_{1}(a / b)$ and hence $1 \in P+(a)$.

The existence of a valuation pair $(V, P)$ with $P$ not a maximal ideal of $V$ shows that there exist valuation rings $V$ with overrings $W$ such that $W$ is not a valuation ring with its set of elements with positive value contained in $V$.

Such an example is given in [1]. We include a sketch for completeness. Let $F$ be a field and consider $F[X, Y]$. Let $G$ be the set of all irreducible polynomials $f(X, Y)$ such that $f(0,0)=0$ and $f(X, 0)$ $\neq 0$. For each $g \in G$, let $Z_{g}$ be an indeterminate over $F$. Let $R=F\left[X, Y, \quad\left\{Z_{0} \mid g \in G\right\}\right]$. Let $I$ be the ideal generated by $\left\{Z_{o} Z_{h} \mid g, h \in G\right\} \cup\left\{g Z_{o} \mid g \in G\right\}$. Let $S=R / I$ and let $Q$ be the ideal generated by the classes of $X$ and $\left\{Z_{\theta} \mid g \in G\right\}$. Then $Q$ is a prime ideal of $S$ and there exists a valuation pair $(V, P)$ of the total quotient ring of $S$ such that $S \subseteq V$ and $P \cap S=Q$. It can be shown that $P$ is not a maximal ideal of $V$.

Next we show that overrings of valuation rings are "close" to valuation rings.

THEOREM 3. Let $(V, P)$ be a valuation pair and let $W$ be an overring of $V$. Then $W$ is contained in a valuation ring which is a large quotient ring of $V$.

Proof. Let $M=\{x \in V \mid x W \subseteq P\}$. Then $M$ is a prime ideal of $W$ and of $V$. Thus $\left(V_{[M]}, M\right)$ is a valuation pair. (To show this note that $M=\{x \in V \mid x s \in M$ for some $s \in V \backslash M\}$.) Now if $x \notin V_{[M]}$, then there exists $s \in M$ such that $x s \in V \backslash M$. Hence there exists $w \in W$ such that $w x s \notin P$. Thus $w x \notin W$ and so $x \notin W$. Therefore $W \subseteq V_{[M]}$.

We can sharpen this result for certain overrings.

THEOREM 4. Let $(V, P)$ be a valuation pair and let $W$ be an overring of $V$. If $W$ is a large quotient ring of $V$, then there exists a valuation ring $U$ which contains $W$ and such that any element in $U$ and not in $W$ is a zero divisor.

Proof. Let $W=V_{[N]}$ for some prime ideal $N$ of $V$. If $W=K$, then $(W, N W)$ is a valuation pair and the theorem is proved. Let $M$ be as in Theorem 3. Then $V_{[M]}$ is a valuation ring containing $W$. If $m \in M \backslash N$ and if $y \in K \backslash W$, then $m y \notin V$, so there exists $p \in P$ such that 
$m y p \in V \backslash P$. Thus $y p \in V_{[N]}=W$ and $m y p \in P$, contrary to fact. Thus $M \subseteq N$. Let $x \in N \backslash M$; then there exists $w \in W$ such that $w x \notin P$. If $x$ is regular, then there exists $q \in V \backslash P$ such that $q / x \in V_{[N]}$ and hence there exists $r \in V \backslash N$ such that $r q / x \in V$. Since $q \in V \backslash P, r / x \in V$; that is, $r \in N$ which is not the case. Thus $x$ is a zero divisor.

Suppose $z$ is an element of $V_{[M]} \backslash W$. Then there exists $p \in P$ such that $z p=q \in V \backslash P$. If $z$ were regular, this would yield $z^{-1} q=p \in V$ so, as $q \in V \backslash P, z^{-1} \in V$. But, $z \notin V_{[N]}=W$ with $z z^{-1} \in V$ implies $z^{-1} \in N$ which implies, as $z^{-1}$ is regular, that $z^{-1} \in M$. Hence, $1=z z^{-1} \in$ $V_{[M]} \cdot M \subseteq M$, a contradiction.

Recall the example of [1] cited after Theorem 2. If $W=V[X / Y]$, then there is no valuation pair $(U, T)$, such that $U$ is a large quotient ring of $V$ containing $W$ and $T \subseteq P$, which has the property that any element in $U$ which is not in $W$ is a zero divisor.

\section{BIBLIOGRAPHY}

1. Monte B. Boisen, Jr. and Max D. Larsen, Overrings of valuation and Prïfer rings, Pacific J. Math. (to appear).

2. N. Bourbaki, Algèbre commutative, Chap. 6, Actualités Sci. Indust., no. 1308, Hermann, Paris, 1964. MR 33 \#2660.

3. Malcolm Griffin, Prïfer rings with zero divisors, J. Reine Angew. Math. 239/ 240 (1969), 55-67. MR 41 \#188.

4. D. K. Harrison, Finite and infinite primes for rings and fields, Mem. Amer. Math. Soc. No. 68 (1966). MR 34 \#7550.

5. Merle E. Manis, Valuations on a commutative ring, Proc. Amer. Math. Soc. 20 (1969), 193-198. MR 38 \#2134.

UNIVERSity OF NEBRASKa, LiNCOLN, NEBRASKa 68508 